\title{
The Evolution of Empty Flowers
}

\author{
Graham Bell \\ Biology Department, McGill University, 1205 Avenue Dr. Penfield, Montreal, \\ Quebec, Canada $H 3 A 1 B 1$
}

(Received 3 January 1985)

\begin{abstract}
This paper describes a simple model intended as a first step towards a quantitative theory of the flower. It divides both flowers and their visitors into two categories: the flowers may either produce a substantial volume of nectar ("secretors") or none ("cheaters"), while the insects either attempt to discriminate between them and enter only secretors ("selectors") or enter any flower encountered ("neglectors"). If the insects can learn the state of the plant population and behave accordingly, then they should all either select or all neglect exclusively; the plants adopt a mixed strategy with each plant producing a similar proportion of cheating flowers. The proportion increases as the time required by the selectors to discriminate between cheaters and secretors increases relative to the time required to extract the nectar. The argument is of general interest because it represents a new theory of nectar concealment. Cheating is known to occur in some plants, but it is not known how widespread the strategy is.
\end{abstract}

The main function of flowers is to attract the insects which import and export pollen grains. The way in which the architecture of the flower permits or enforces the correct reception of the pollen onto the stigma and its deposition from the anthers has been the subject of a large literature over the last century (see Knuth (1906) for a wealth of detail), especially in relation to the means for securing crossfertilization. Despite the volume of this descriptive work, however, there is virtually no quantitative theory of pollination from the plant's point of view. An increased number of insect visits must generally result in a greater proportion of the ovules being fertilized, and more particularly in a greater proportion of the pollen being dispersed. At the same time, further increases in the number of visits must sooner or later yield diminishing returns: if ten insect visits are sufficient to remove three-quarters of the pollen or to fertilize three-quarters of the ovules then twice as many visits cannot double the benefit to the plant. Plants should therefore evolve so as to optimize their expenditure on flowers, by maximizing the number of fertilizations obtained per unit of secondary allocation to floral attractants and rewards.

The purpose of this note is not to develop a general quantitative theory of flowers, but rather to describe one special case, in which a dichotomy in floral allocation seems to arise naturally from the interaction between flowers and their visitors, and whose validity can be investigated by simple measurements of flowers in natural populations.

Suppose that some plants ("secretors") have expensive nectar-bearing flowers whilst others ("cheaters") have empty flowers which are cheaper to make. The 
insects which visit these flowers are also of two kinds: "selectors", which visit only secretors but can distinguish them from cheaters only at the cost of an increase in the length of time needed for each visit, and "neglectors", which enter all the flowers they encounter. In their simplest form, these rules describe a cyclical game (Fig. 1)

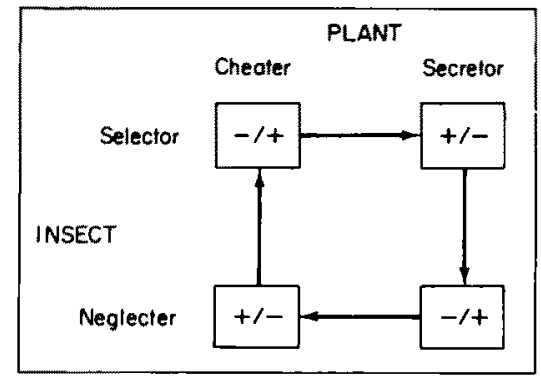

FIG. 1. Cheating as a strategy which leads to a cyclical game. The payoff to the plant is indicated before the diagonal in each cell and the payoff to the insect after: "+" indicates that relevant strategy is better than the alternative and "- " that it is worse, when the populations of plants and insects are nearly fixed for the two relevant pure strategies.

analogous to Dawkins' (1976) "battle of the sexes". Suppose that the plant population at some point in time contains a large proportion of cheaters, while the insects are mostly selectors. The selector strategy benefits the insects, since they can avoid the many cheaters, but most of the plants are never visited. The plants should therefore switch to a secretor strategy; they then receive more visits, but the insects are now paying an unnecessary cost in terms of the time required to discriminate between cheaters and secretors, which lowers their net rate of energy acquisition. The insects should then become neglectors, lowering the payoff to the plants from expensive secretor flowers and so favouring cheaters. When the cheaters again prevail in the plant population the insects do better by being selectors, and the cycle is complete.

We can easily formalize this game. Let the average number of visits received by a flower be $V$; a visit may or may not result in pollination, depending on whether or not the insect enters the flower-neglectors always enter, but selectors enter only secretors. Each entry results in the removal of a quantity $G$ of pollen. In practice the quantity of pollen removed may be a decreasing function of the quantity remaining from previous visits, and visits serve to fertilize ovules as well as to remove pollen. So long as the number of fertilizations obtained (through pollen or ovules) is an increasing function of the number of visits, however, the argument given here is qualitatively valid. Each secretor flower produces a quantity $N$ of nectar, which is related to the total cost of the flower by a constant $k$ : a cheater costs $k N$ units of resource to make, while the cost of a secretor is $(1+k) N$. Among the insects, some variables take the same value for neglectors and selectors: the rate of encounter with flowers is $\lambda$, the handling time per flower is $H$, and the nectar reward from secretors is $R$. The selectors, however, spend a certain length of time $D$ in ascertaining whether each flower encountered is a cheater or a secretor. 
When each plant produces only one kind of flower, the payoff from each pure strategy is

$$
\begin{aligned}
\text { cheater: } & V(1-p) G / k N \\
\text { secretor: } & V G /(1+k) N
\end{aligned}
$$

where $p$ is the frequency of selectors among the insects, and the payoff is in terms of the total number of pollen grains exported per plant. If each insect likewise plays only one of the two pure strategies, the payoffs are

$$
\begin{aligned}
\text { selector: } & (1-q) R /\left[\lambda^{-1}+H(1-q)+D\right] \\
\text { neglector: } & (1-q) R /\left[\lambda^{-1}+H\right]
\end{aligned}
$$

where $q$ is the frequency of cheaters among the plants, and the payoff is in terms of the quantity of energy harvested per unit time; the form of the expressions is standard in the theory of optimal diets (see Charnov, 1972).

These four payoffs describe a cyclical game. If the plant and insect strategies are both determined by alleles at a single haploid locus, then it is easy to show that gene frequencies in both populations cycle through time, usually with long period and large amplitude, as the result of the time-lagged frequency-dependence built into the model. However, such a situation is quite unrealistic. In the first place, if plants play a pure strategy then the insects could quickly learn to leave a plant after encountering a single empty flower. Secondly, it is assumed that plants and insects play alternate moves; but in practice insects can quickly learn to respond appropriately to the reward schedule offered by the plants (see Heinrich, 1978), whereas only a genetic response is available to the plants. A more realistic model, therefore, would incorporate a mixed strategy for the plants and learning by the insects.

To calculate the payoff for a plant using strategy $(q)$, suppose that it possesses a total of $S$ units of resource, and produces $C$ cheating flowers. It can then produce $(S-C k N) /(1+k) N$ secreting flowers, so that the total payoff to the plant is

$$
E(q)=C V(1-p) G+V G(S-C k N) /(1+k) N
$$

Since the frequency of cheating flowers on the plant is by definition $q=C /[C+$ $(S-C k N) /(1+k) N]=(1+k N) /(1+S / C)$, we have $C=q S /(1+k-q) N$ to substitute into the expression for $E(q)$, yielding

$$
E(q)=\text { constant } \times(1-p q) /(1+k-q) \text {. }
$$

The payoff to an insect playing strategy $(p)$ is

$$
F(p)=p(1-q) R /\left[\lambda^{-1}+H(1-q)+D\right]+(1-p)(1-q) R /\left(\lambda^{-1}+H\right),
$$

so that $\partial F(p) / \partial p<0$ if $q>D / H$. Since the insects can learn very quickly relative to the generation time of the plants, the strategy used by the insects should be

$$
\begin{cases}p=0 & \text { if } q<D / H \\ p=1 & \text { if } q>D / H\end{cases}
$$

I have simulated this interaction by allowing each of the 101 equidistant phenotypes 
in $q \subseteq(0,1)$ to be determined by a different allele at a haploid locus. Both plant and insect populations change through time, the insect population flipping from $p=0$ to $p=1$ or vice versa according to the average state of the plant population, while the average state of the plants responds in a continuous fashion to the strategy of the insects. However, the fluctuations of $q$ quickly settle down to an irregular oscillation of very small amplitude centred around $q=D / H$. Moreover, extreme phenotypes ( $q$ very small or very large) are rapidly eliminated, and the plant population eventually comes to comprise only a narrow range of phenotypes close to $D / H$. In similar models with diploid genetics, or in which the insects adopt an indeterminate mixed strategy when $q$ is very close to $D / H$, plant strategy is even more stable.

The major conclusion from this argument is that we expect plants to adopt a mixed strategy $q \sim D / H$, with all individuals producing a similar substantial fraction of cheap flowers virtually devoid of nectar if the time required by the insects to discriminate between cheating and secreting flowers is substantial relative to the time required to extract the nectar from a secretor. It is predicted, therefore, that plants whose nectar is deeply concealed within the flower will often bear cheating flowers; and that the frequency of cheats will rise as the discrimination time increases relative to the handling time.

The standing crop of nectar in flowers is often measured during foraging studies, and in many cases a standard deviation is cited, though the frequency distribution of nectar volume is rarely given. If cheating flowers are common, we expect that many flowers will contain an abundance of nectar whilst others bear none. Unfortunately, such standing-crop measurements cannot be used to test the theory since a similar pattern will be created by the activity of insects even in the absence of cheaters (Brink, 1982; Pleasants \& Zimmerman, 1983). If insects visit flowers at random then the arrival of bees at flowers is a Poisson process, and for any given flower the waiting time until the next visit will be exponentially distributed. Since nectar volume usually increases more or less linearly after depletion (Cruden et al., 1983), the standing crop of nectar will also become exponentially distributed once insect visits have got under way. The frequency distribution of standing crops will therefore have a mode at very small nectar volumes, even if all flowers secrete nectar. This simple hypothesis is readily tested using the large data set supplied by Cruden et al. (1983), since for an exponential distribution the standard deviation is equal to the mean. It turns out to be false (Fig. 2), but the variance of the standing crop is actually less than that predicted on the basis of random visits to flowers. I suggest that this effect may be due to a preference of visitors for flowers whose nectar volume is great enough to yield a rate of reward equal to or exceeding that of the average of its neighbours, as predicted by the marginal value theorem of Charnov (1976); but at all events the measurement of standing crop gives no support to the argument developed here, and perhaps cannot supply a decisive test of the existence of cheating flowers.

The crucial measurement is therefore the frequency distribution of nectar production rates, got by measuring nectar volume in bagged flowers. The only such data I have found in the literature are the frequency distributions given by Feinsinger 


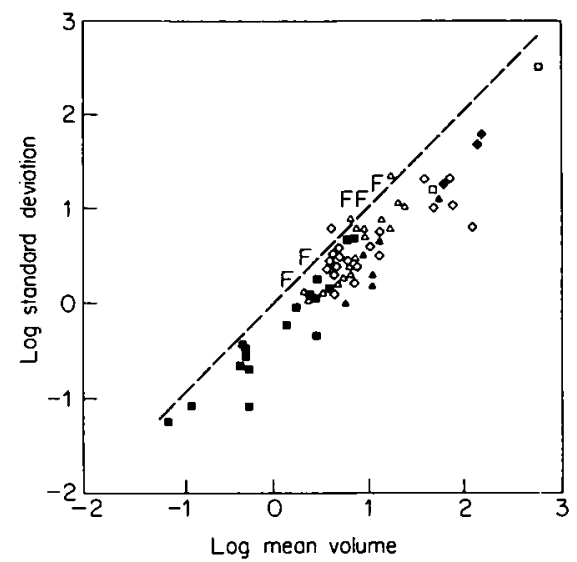

FIG. 2. The relationship between the mean and the standard deviation of nectar standing crop. Plotted points are data from Cruden et al. (1983), different symbols indicating different pollinators: $\bullet$ bat; $\diamond$ hawkmoth; $\Delta$ hummingbird; $\dot{\Delta}$ sunbird; $\square$ "oriole-starling" guild; bees, butterflies and small moths. The broken line has unit slope and represents the expected outcome of an exponential probability distribution of nectar volume. The " $F$ " symbols are Feinsinger's data for bagged flowers of five tropical plants, included on the diagram for comparison with the standing crop data.

(1978) for five tropical plants visited by hummingbirds. In all five cases his figures show a large proportion of the flowers producing little or no nectar, whilst a few produce very large quantities. Feinsinger interprets this pattern as a device which forces the hummingbirds to visit more flowers in order to obtain an adequate diet, and the model developed in this paper can be taken as an extension and a confirmation of his reasoning.

Feinsinger's data establishes the existence of cheating flowers, but their generality remains unknown, and other data are less conclusive. Brink \& deWet (1980) found that nectar production was very variable within and between populations of a species of Aconitum, and some of their samples included substantial numbers of nonsecreting flowers. However, this variance was largely accounted for by differences in flower age within populations and by differences in mean nectary depth between populations; moreover, populations with more deeply concealed nectar had lower frequencies of empty flowers, the reverse of the pattern predicted by the model.

If the nectar is so effectively concealed that discrimination time exceeds handling time, then plants should produce cheating flowers exclusively. The argument then becomes too complex for a simple model to represent, since the pollinators could learn to avoid such plants entirely and forage on a different species. A pure cheating strategy can be sustained only if the time required to discriminate between species is prohibitively long. I suggest that this extension of the basic model provides an interpretation of cases such as Cephalanthera rubra, an orchid whose large and conspicuous flowers secrete no nectar but closely resemble those of nectariferous Campanula (Nilsson, 1983), or the similar relationship between Calopogon pulchellus and Pogonia ophioglossoides (Krebs \& Davies, 1981).

The traditional interpretation of nectar concealment has been that it enforces pollinator constancy and therefore the correct reception and deposition of pollen 
in morphologically complex flowers, an idea dating back at least to Muller (1883). A narrow range of pollinator species is certainly a consequence of nectar concealment, and leads in turn to the evolution of complex floral architecture. The hypothesis I have developed above, however, suggests that nectar concealment is more fundamentally a device which permits economy in allocation to flowers by facilitating the evolution of cheating. Further studies of the variability of nectar production in bagged flowers would be of great interest.

This work was supported in part by an Operating Grant from the National Science and Engineering Research Council of Canada.

\section{REFERENCES}

BrINK, D. (1982). Oecologia 52, 292.

BRINK, D. \& DE WET, J. M. (1980). Oecologia 47, 160.

Charnov, E. L. (1972). Ph.D. thesis, University of Washington.

Charnov, E. L. (1976). Theor. Pop. Biol. 9, 129.

Cruden, R. W., Hermann, M. H. \& Peterson, S. (1983). In: The Biology of Nectaries. (Bentley, B.

\& Elias, T. eds). pp. 80-125, New York: Columbia University Press.

Dawkins, R. (1976). The Selfish Gene. Oxford: Oxford University Press.

FeINSINGER, P. (1978). Ecol Monogr. 48, 269.

HEINRICH, B. (1978). Bumble-bee Economics. Cambridge, Mass.: Harvard University Press.

KNUTH, P. (1906). Handbook of Flower Pollination, 3 vols. Oxford: Clarendon Press.

KREBS, J. R. \& DAVIES, N. B. (1981). An Introduction to Behavioural Ecology. Sunderland, Massachusetts:

Sinauer.

MULLER, H. (1983). The Fertilization of Flowers. London: MacMillan.

Nilsson, L. A. (1983). Nature 305, 799.

Pleasants, J. M. \& Zimmerman, M. (1983). Oecologia 57, 412. 